\title{
Why Are Firms Sometimes Unwilling to Reduce Costs?
}

\author{
X. Henry Wang and Jingang Zhao \\ University of Missouri-Columbia University of Saskatchewan \\ January 2007
}

\begin{abstract}
This paper establishes three new results for multiproduct oligopolies: 1) it presents the first explicit expression of Nash equilibria for asymmetric multiproduct oligopolies; 2) it shows that reducing a multiproduct firm's cost in Bertrand oligopolies will reduce its profits if the cost-reducing unit is sufficiently small; and 3) it demonstrates that a multiproduct firm has no incentive to eliminate a product whose sales are zero. Because a single-product firm whose sales are zero is indifferent between exiting and staying, and its cost reductions always increase its profits, our results are unique to the multiproduct firm, and they suggest that extending oligopoly studies from a single product to multi-products could be as significant as the extension of calculus from a single variable to multi-variables.

Keywords: Effect of cost reduction, multiproduct oligopoly, price competition, quantity competition

JEL Classification Numbers: C63, D43, L13
\end{abstract}

\section{Introduction}

We study multiproduct oligopolies with asymmetric costs in both price and quantity competition. We first derive closed-form expressions for multiproduct Bertrand and 
Cournot equilibria with asymmetric linear costs. To our knowledge, such equilibrium expressions have not been reported in the existing literature. The few available expressions were limited either to duopolies (Bulow, Geanakoplos and Klemperer [1985]; and Lal and Matutes [1989]) or to symmetric product lines (Grossmann [2003]), and most other previous studies of multiproduct oligopolies were completed without the general expressions. ${ }^{1}$ In the merger literature, Deneckere and Davidson ([4], 1985) provided expressions for postmerger Bertrand equilibria with zero costs, which are identical to a special class of multiproduct Bertrand equilibria. Because postmerger Bertrand equilibria from an arbitrary coalition structure with asymmetric costs are identical to a general multiproduct Bertrand equilibrium, their two-decade old assessment that "it is no longer possible to write analytical expressions for equilibrium payoffs" ([4], p. 481) was still an accurate account of today's literature prior to this study. The unavailability of a general expression for multiproduct equilibria was caused perhaps by the unavailability of the involved tools or inverse matrices for solving the problem. Indeed, without using the new inverse matrices in Zhao and Howe (2004), we would never have been able to obtain the equilibrium expressions.

Next, we evaluate the effects of technological innovation. We show that reducing a multiproduct firm's cost could reduce its profits in price competition, but will always increase its profits in quantity competition. The negative relationship between cost reduction and profit explains why some firms are unwilling to reduce cost or adopt new technologies, even if such cost reduction and technology adoption are free. Although previous studies have shown how small cost reductions could reduce social welfare, this study is the first to show how small cost reductions could reduce the cost-reducing firm's profits. ${ }^{2}$

At first glance, the negative profit effect of cost reduction is counterintuitive, be-

\footnotetext{
${ }^{1}$ For example, Harrington (1987) studied collusion, Zhang and Zhang (1996) studied stability, and Johnson and Myatt (2003) studied quality competition, all in multiproduct Cournot Oligopolies; Cabral and Villas-Boas (2001) studied product externality in multiproduct Bertrand oligopolies; and Goldberg (1995) estimated the multiproduct market of the US automobile industry.

${ }^{2}$ For recent works on the welfare effects of cost reduction, see Février and Linnemer (2004) and Smythe and Zhao (2006). Schelling ([13], 1960) showed the possibility that an exogenous increase in a player's payoff function could reduce his equilibrium payoff. However, it was not known, prior to this study, if his game example ([13], p. 158) could arise from a general economic problem.
} 
cause conventional theory of the firm holds that a firm should always benefit from cost reductions and technological innovations if the cost of obtaining such improvement is zero or low. As readers will see, the negative effect of cost reduction on a multiproduct Bertrand firm's profits is the confluence of three forces. One force is strategic complementarity in price competition. If firms' choices are strategic substitutes (such as in Cournot competition), the profit effects of cost reductions will always be positive. Another force is strategic interaction between the multiproduct firm and other firms. If there are no strategic interactions (such as in a monopoly), cost reductions will always increase profits. The other force is output reallocation: a cost reduction in one unit increases the production in this unit, but decreases productions in all other units inside the firm. These forces work to raise the profit of the cost-reducing unit but lower the profits of all other units inside the multiproduct firm. When the cost-reducing unit is small, its profit gain is outweighed by profit losses from all other units, leading to a reduction in the multiproduct firm's total profits. Precisely, we derive two closed-form critical levels for identifying the profit effects of cost reduction: 1) critical output share: a small reduction in the marginal cost of a small unit reduces the multiproduct firm's profits if and only if its output share in the multiproduct firm is below the critical level; 2) critical size of cost reduction: a large reduction in the marginal cost of a small unit reduces the multiproduct firm's profit if and only if the size of the cost reduction is below the critical level.

Finally, we show that a multiproduct firm has no incentive to eliminate a product whose sales are zero. Because a single-product firm whose sales are zero is indifferent between exiting and staying, and its cost reductions always increase its profits, our results are unique to the multiproduct firm; they support the belief that extending oligopoly studies from a single product to multi-products could be as significant as the extension of calculus from a single variable to multi-variables.

The remainder of the paper is organized as follows. Section 2 describes the model and provides the closed-form expressions for multiproduct Bertrand and Cournot equilibria in asymmetric linear oligopolies. Section 3 studies the profit effects of a Bertrand firm's cost reduction and product closing, and section 4 studies the same 
issues for a Cournot firm. Section 5 concludes the paper, and the appendix provides all proofs.

\section{Calculation of Bertrand and Cournot Equilibria}

A linear Bertrand oligopoly with $n$ goods, or the Bertrand-Shubik model, is defined by $n$ demand and $n$ cost functions (see Bertrand [1883], Shubik [1980]):

$$
q_{i}(p)=V-p_{i}-\gamma\left(p_{i}-\bar{p}\right), C_{i}\left(q_{i}\right)=c_{i} q_{i}, i \in N=\{1, \ldots, n\}
$$

where $V>0$ is the common intercept of demand functions, $p_{i}$ is the price of good $i, p=\left(p_{1}, \ldots, p_{n}\right)^{\top}$ is the price vector, $\gamma \geq 0$ is the substitutability parameter, $\bar{p}=$ $\left(\sum_{j=1}^{n} p_{j}\right) / n$ is the average price, and $c_{i}$ is the constant marginal (or average) cost of producing good $i$.

These goods are independent if $\gamma=0$, and they become closer substitutes as $\gamma$ increases toward infinity. They can be supplied by a multiproduct monopoly, by $n$ single-product firms, or by $k$ multiproduct firms $(1<k<n)$, which correspond, respectively, to three classes of market structures (i.e., partitions of $N$ ): $i$ ) a multiproduct monopoly, or the coarsest partition $\Delta_{m}=\{N\}$; ii) an oligopoly with single-product firms, or the finest partition $\Delta_{0}=\{1,2, \ldots, n\}$; and iii) an oligopoly with multiproduct firms, or a general partition $\Delta=\left\{S_{1}, S_{2}, \ldots, S_{k}\right\}$ of $N$ (i.e., $S_{i} \neq \varnothing$, $S_{i} \cap S_{j}=\varnothing$, all $i \neq j$, and $\left.\cup S_{j}=N\right)$, where $1<k<n$, and for each $j=1, \ldots, k$, firm $S_{j}$ produces $\left|S_{j}\right|=n_{j}$ products (i.e., $\sum_{j=1}^{k} n_{j}=n$ ).

For each firm $S \in \Delta$, let $p_{S}=\left\{p_{i} \mid i \in S\right\}$ and $q_{S}=\left\{q_{i} \mid i \in S\right\}$ denote its price and output vectors, and $p_{-S}=\left\{p_{i} \mid i \in N \backslash S\right\}$ denote the vector of other firms' prices. Then, for each $p=\left(p_{S}, p_{-S}\right)=\left(p_{1}, \ldots, p_{n}\right)^{\top}$, the profit of a firm $S \in \Delta$ is given by

$$
\pi_{S}(p)=\pi_{S}\left(p_{S}, p_{-S}\right)=\sum_{i \in S} \pi_{i}(p)=\sum_{i \in S} q_{i}(p)\left(p_{i}-c_{i}\right)
$$

and the Bertrand equilibrium (or Nash equilibrium or strategic equilibrium) is a price 
vector $p^{*}=\left\{p_{S}^{*} \mid S \in \Delta\right\}=\left(p_{1}^{*}, \ldots, p_{n}^{*}\right)^{\top}$ such that for each firm $S \in \Delta, p_{S}^{*}$ is its best response to others' price vector $p_{-S}^{*}$, or that each $p_{S}^{*}$ solves $\operatorname{Max}\left\{\pi_{S}\left(p_{S}, p_{-S}^{*}\right) \mid p_{S}\right\}$, where $\pi_{S}\left(p_{S}, p_{-S}\right)$ is given in $(2)$.

Throughout the paper we assume that a unique equilibrium always exists. Under this assumption, the Bertrand equilibrium for $\Delta=\left\{S_{1}, S_{2}, \ldots, S_{k}\right\}$ is the solution to the following $k$ sets of first-order conditions:

$$
\text { For each } S \in \Delta, \text { and all } i \in S, \frac{\partial \pi_{S}\left(p_{S}, p_{-S}\right)}{\partial p_{i}}=0
$$

Rearranging (3) leads to the following properties on the equilibrium markups: for each firm $j=1, \ldots, k$, or each $S_{j} \in \Delta$ with $\left|S_{j}\right|=n_{j}$,

$$
\begin{aligned}
(1+\gamma)\left(p_{i}^{*}-c_{i}\right) & =q_{i}^{*}+\frac{n_{j} \gamma\left(\bar{p}_{S_{j}}^{*}-\bar{c}_{S_{j}}\right)}{n}, \text { all } i \in S_{j}, \\
\frac{\bar{p}_{S j}^{*}-\bar{c}_{S_{j}}}{\bar{q}_{S_{j}}^{*}} & =\frac{n}{n(1+\gamma)-n_{j} \gamma}, \text { and } \\
\frac{p_{j}^{*}-c_{j}}{q_{j}^{*}} & =\frac{n}{n(1+\gamma)-\gamma}, \text { for all } j \text { with } n_{j}=1 .
\end{aligned}
$$

where $\bar{p}_{S_{j}}^{*}=\sum_{i \in S_{j}} p_{i}^{*} / n_{j}, \quad \bar{c}_{S_{j}}=\sum_{i \in S_{j}} c_{i} / n_{j}$, and $\bar{q}_{S_{j}}^{*}=\sum_{i \in S_{j}} q_{i}^{*} / n_{j}$ are firm $j$ 's average price, average marginal cost, and average supply, respectively. By (6), all single-product firms have the same markup/supply ratio, $n /(n(1+\gamma)-\gamma)$. And by (4) and (5), different units in a multiproduct firm may have different markup/supply ratios, and a multiproduct firm has a larger average-markup/average-supply ratio than does a single-product firm (i.e., $\left.n /\left(n(1+\gamma)-n_{j} \gamma\right)>n /(n(1+\gamma)-\gamma)\right)$. 
Let $\delta, a, b, c>0$ and $d=\left\{d_{S} \mid S \in \Delta\right\}=\left(d_{1}, \ldots, d_{n}\right)^{\top}$ be defined as

$$
\begin{aligned}
\delta & =n(1+\gamma)-\gamma ; \\
a & =2 \delta, b=2 \gamma, c=\gamma ; \text { and for each } S \in \Delta, \\
d_{S} & =\left\{d_{i} \mid i \in S\right\}, \text { where } d_{i}=n V+\delta c_{i}-\gamma \Sigma_{j \in S \backslash i} c_{j}, \text { all } i \in S .
\end{aligned}
$$

Then, the first-order conditions in (3) can be rearranged as

$$
A p=d, \text { where } A=A_{n \times n}=\left(\begin{array}{cccc}
A_{11} & A_{12} & \cdots & A_{1 k} \\
A_{21} & A_{22} & \cdots & A_{2 k} \\
\vdots & \vdots & \ddots & \vdots \\
A_{k 1} & A_{k 2} & \cdots & A_{k k}
\end{array}\right)
$$

is an $n \times n$ matrix with $k^{2}$ blocks whose entries are: 1) for $j=1, \ldots, k, A_{j j}$ is an $n_{j} \times n_{j}$ symmetric matrix such that all its main diagonal entries are $a$, and all its off-diagonal entries are $-b$, where $n_{j}=\left|S_{j}\right|$ is the number of goods produced by firm $\left.S_{j} \in \Delta ; 2\right)$ for all $i \neq j, A_{i j}$ is an $n_{i} \times n_{j}$ matrix whose entries are all $-c$; and 3) $\sum_{j=1}^{k} n_{j}=n$.

In order to analyze how cost reductions or technological changes affect equilibrium profits, we focus on the class of Bertrand oligopolies with a single multiproduct firm given by $\Delta=\{S, m+1, \ldots, n\}=\{\{1, \ldots, m\}, m+1, \ldots, n\}$. Due to the complexity of the problem (readers could get a taste of such complexity by examining the detailed formulae in (39)), studying the general case in (8) or its solution in (22) would deviate from our emphasis on cost reductions. 
Given $\Delta=\{S, m+1, \ldots, n\}$, the first-order conditions in (3) become

$$
\frac{\partial \sum_{k \in S} \pi_{k}}{\partial p_{i}}=0, \text { all } i \in S ; \text { and } \frac{\partial \pi_{j}(p)}{\partial p_{j}}=0, \text { all } j \in N \backslash S,
$$

which is a special case of (8).

The inverse demands of the Bertrand-Shubik demand system given in (1) are:

$$
p_{i}(q)=p_{i}\left(q_{1}, \ldots, q_{n}\right)=V-q_{i}+\frac{\gamma}{1+\gamma}\left(q_{i}-\bar{q}\right),
$$

where $\bar{q}=\left(\sum_{j=1}^{n} q_{j}\right) / n$ is the industry's average output. Now, firm $i$ 's profit function becomes $\pi_{i}(q)=\left(p_{i}(q)-c_{i}\right) q_{i}$. A Cournot oligopoly with $k$ multiproduct firms is also defined by the partition $\Delta=\left\{S_{1}, S_{2}, \ldots, S_{k}\right\}$ with $1 \leq k<n$. For each firm $S \in \Delta$, its profit is given by $\pi_{S}(q)=\pi_{S}\left(q_{S}, q_{-S}\right)=\sum_{i \in S} \pi_{i}(q)=\sum_{i \in S}\left(p_{i}(q)-c_{i}\right) q_{i}$. The Cournot equilibrium is an output vector $q^{C *}=\left\{q_{S}^{C *} \mid S \in \Delta\right\}=\left(q_{1}^{C *}, \ldots, q_{n}^{C *}\right)^{\top}$ such that for each $S \in \Delta, q_{S}^{C *}$ is its best response to others' output vector $q_{-S}^{C *}$, or that each $q_{S}^{C *}$ solves $\operatorname{Max}\left\{\pi_{S}\left(q_{S}, q_{-S}^{C *}\right) \mid q_{S}\right\}$. Under the assumption for a unique equilibrium, the Cournot equilibrium for $\Delta=\left\{S_{1}, S_{2}, \ldots, S_{k}\right\}$ is the solution to the following $k$ sets of first-order conditions:

$$
\text { For each } S \in \Delta \text {, and all } i \in S, \frac{\partial \pi_{S}\left(q_{S}, q_{-S}\right)}{\partial q_{i}}=0 \text {. }
$$

Let $\bar{a}, \bar{b}, \bar{c}>0$ and $\bar{d}=\left(\bar{d}_{1}, \ldots, \bar{d}_{n}\right)^{\top}$ be defined as

$$
\begin{aligned}
\bar{a} & =2(n+\gamma), \bar{b}=-2 \gamma, \bar{c}=-\gamma, \\
\bar{d}_{i} & =n(1+\gamma)\left(V-c_{i}\right), \text { all } i \in N .
\end{aligned}
$$


Then, the first-order conditions in (11) can be rearranged as

$$
B q=\bar{d}, \text { where } B=B_{n \times n}=\left(\begin{array}{cccc}
B_{11} & B_{12} & \cdots & B_{1 k} \\
B_{21} & B_{22} & \cdots & B_{2 k} \\
\vdots & \vdots & \ddots & \vdots \\
B_{k 1} & B_{k 2} & \cdots & B_{k k}
\end{array}\right)
$$

is identical to $A$ in (8) except that the constants $a, b, c$, and $d$ in (7) are replaced by $\bar{a}, \bar{b}, \bar{c}$, and $\bar{d}$ in $(12)$.

As in the Bertrand model, we focus on Cournot oligopolies with a single multiproduct firm given by $\Delta=\{S, m+1, \ldots, n\}$, whose first-order conditions are:

$$
\frac{\partial \sum_{j \in S} \pi_{j}}{\partial q_{i}}=0, \text { all } i \in S ; \text { and } \frac{\partial \pi_{i}(q)}{\partial q_{i}}=0 \text {, all } i \in N \backslash S \text {. }
$$

Rearranging the above first-order conditions leads to:

$$
\begin{aligned}
(1+\gamma)\left(p_{i}-c_{i}\right) & =q_{i}+\frac{m \gamma \bar{q}_{S}}{n}, \text { all } i \in S, \\
\frac{\bar{p}_{S}-\bar{c}_{S}}{\bar{q}_{S}} & =\frac{n+m \gamma}{n(1+\gamma)}, \text { and } \\
\frac{p_{i}-c_{i}}{q_{i}} & =\frac{n+\gamma}{n(1+\gamma)}, \text { all } i \in N \backslash S,
\end{aligned}
$$

so the markups in a Cournot model are analogous to those in the Bertrand model in (4-6): single-product firms have the same markup/supply ratio, different units in the multiproduct firm might have different markup/supply ratios, and the multiproduct firm's average-markup/average-supply ratio is larger.

Applying the general inverse in (39) to (13-14) leads to the following Cournot equilibrium with multiproduct firms: 
Proposition 1 Consider the Cournot oligopoly in (10). (i) Given $\Delta=\left\{S_{1}, S_{2}, \ldots, S_{k}\right\}$, its Cournot equilibrium is given by

$$
q^{C *}=B^{-1} \bar{d}=\left(\begin{array}{cccc}
W_{11} & W_{12} & \cdots & W_{1 k} \\
W_{21} & W_{22} & \cdots & W_{2 k} \\
\vdots & \vdots & \ddots & \vdots \\
W_{k 1} & W_{k 2} & \cdots & W_{k k}
\end{array}\right)\left(\begin{array}{c}
\bar{d}_{S_{1}} \\
\bar{d}_{S_{2}} \\
\vdots \\
\bar{d}_{S_{k}}
\end{array}\right)
$$

where $B^{-1}=W$ is the same as that of (39), except that the constants $a, b$, $c$, and $d$ in (7) are replaced by $\bar{a}, \bar{b}, \bar{c}$, and $\bar{d}$ in (12).

(ii) Given $\Delta=\{S, m+1, \ldots, n\}$, the Cournot equilibrium in (18) becomes: for each unit $i \in S$, and each single-product firm $j \in N \backslash S$,

$$
\begin{aligned}
q_{i}^{C *}= & \frac{n(1+\gamma)(2 n+\gamma) V}{\omega_{1}}+\frac{(1+\gamma)(4 n+(n-m+2) \gamma) m \gamma \bar{c}_{S}}{2 \omega_{1}} \\
& +\frac{n(1+\gamma)(n-m) \gamma \bar{c}_{-S}}{\omega_{1}}-\frac{(1+\gamma) c_{i}}{2} ; \text { and } \\
q_{j}^{C *}= & \frac{n(1+\gamma)(2 n+m \gamma) V}{\omega_{1}}+\frac{n(1+\gamma) m \gamma \bar{c}_{S}}{\omega_{1}} \\
& \quad+\frac{n(n-m)(1+\gamma)(2 n+m \gamma) \gamma \bar{c}_{-S}}{(2 n+\gamma) \omega_{1}}-\frac{n(1+\gamma) c_{j}}{2 n+\gamma},
\end{aligned}
$$

where $\bar{c}_{S}=\sum_{k \in S} c_{k} / m, \bar{c}_{-S}=\sum_{k \notin S} c_{k} /(n-m)$, and $\omega_{1}>0$ is given by

$$
\omega_{1}=\omega_{1}(n, m, \gamma)=m(n-m+2) \gamma^{2}+2 n(n+m+1) \gamma+4 n^{2} .
$$

Similarly, one can show that the Bertrand equilibrium for $\Delta=\left\{S_{1}, S_{2}, \ldots, S_{k}\right\}$ in (1) is given by 


$$
p^{*}=A^{-1} d=\left(\begin{array}{cccc}
U_{11} & U_{12} & \cdots & U_{1 k} \\
U_{21} & U_{22} & \cdots & U_{2 k} \\
\vdots & \vdots & \ddots & \vdots \\
U_{k 1} & U_{k 2} & \cdots & U_{k k}
\end{array}\right)\left(\begin{array}{c}
d_{S_{1}} \\
d_{S_{2}} \\
\vdots \\
d_{S_{k}}
\end{array}\right)
$$

where the inverse matrix $A^{-1}=U$ in (39) is defined by parameters in (7-8). As a special case, the Bertrand equilibrium for $\Delta=\{S, m+1, \ldots, n\}$ are: for each unit $i \in S$, and each single-product firm $j \in N \backslash S$,

$$
\begin{aligned}
p_{i}^{*}= & \frac{n(2 n(1+\gamma)-\gamma) V}{\omega_{2}}+\frac{\gamma^{2} m(n-m) \bar{c}_{S}}{2 \omega_{2}}+\frac{\gamma(n(1+\gamma)-\gamma)(n-m) \bar{c}_{-S}}{\omega_{2}}+\frac{c_{i}}{2}, \\
p_{j}^{*}= & \frac{n(2 n(1+\gamma)-m \gamma) V}{\omega_{2}}+\frac{\gamma m(n(1+\gamma)-m \gamma) \bar{c}_{S}}{\omega_{2}} \\
& +\frac{\gamma(n(1+\gamma)-\gamma)(2 n(1+\gamma)-m \gamma)(n-m) \bar{c}_{-S}}{(2 n(1+\gamma)-\gamma) \omega_{2}}+\frac{(n(1+\gamma)-\gamma) c_{j}}{2 n(1+\gamma)-\gamma},
\end{aligned}
$$

where $\omega_{2}=\omega_{2}(n, m, \gamma)>0$ is given by

$$
\omega_{2}(n, m, \gamma)=\gamma^{2}(n-m)(m+2 n-2)+2 n \gamma(3 n-m-1)+4 n^{2} .
$$

It is useful to note that the above closed-form expressions for Cournot and Bertrand equilibria with multiproduct firms are identical to the corresponding postmerger equilibria for mergers with no synergy in linear differentiated oligopolies with single-product firms. To our knowledge, such general expressions have not been reported in the existing literature. ${ }^{3}$ We hope that other scholars will find them useful in extending previous studies in single-product oligopoly to multiproduct oligopolies.

\footnotetext{
${ }^{3}$ The only exception is the Bertrand equilibrium (23-24) for $\Delta=\{S, m+1, \ldots, n\}$, which is identical to the postmerger equilibrium reported in Zhao and Howe (2004); with $c_{j}=0$ for all $j,(23-24)$ are identical to the postmerger equilibrium with zero costs reported in Deneckere and Davidson (1985, p. 475).
} 
In the next two sections, we will conduct the comparative statics analysis of the above Cournot and Bertrand equilibria.

\section{Cost Reductions in Price Competition}

Plugging the Bertrand equilibrium in (23-24) into the demand (1) and simplifying, one obtains the equilibrium products as below: for each $i \in S$ and $j \in N \backslash S$,

$$
\begin{aligned}
q_{i}^{*}= & \frac{(2 n(1+\gamma)-\gamma)(n(1+\gamma)-m \gamma) V}{\omega_{2}}+\frac{\gamma(n(1+\gamma)-\gamma)(n(1+\gamma)-m \gamma)(n-m) \bar{c}_{-S}}{n \omega_{2}} \\
& +\frac{\gamma\left[\gamma^{2}(3 n-2)(n-m)+\gamma n(7 n-3 m-2)+4 n^{2}\right] m \bar{c}_{S}}{2 n \omega_{2}}-\frac{(1+\gamma) c_{i}}{2}, \\
q_{j}^{*}= & \frac{(2 n(1+\gamma)-m \gamma)(n(1+\gamma)-\gamma) V}{\omega_{2}}+\frac{\gamma m(n(1+\gamma)-m \gamma)(n(1+\gamma)-\gamma) \bar{c}_{S}}{n \omega_{2}} \\
& +\frac{\gamma(n(1+\gamma)-\gamma)^{2}(2 n(1+\gamma)-m \gamma)(n-m) \bar{c}_{-S}}{n(2 n(1+\gamma)-\gamma) \omega_{2}}-\frac{(1+\gamma)(n(1+\gamma)-\gamma) c_{j}}{2 n(1+\gamma)-\gamma},
\end{aligned}
$$

which lead to the following equilibrium profits:

$$
\begin{aligned}
\pi_{S}^{*} & =\sum_{i \in S}\left(p_{i}^{*}-c_{i}\right) q_{i}^{*}=\frac{m(n(1+\gamma)-m \gamma)\left(\bar{p}_{S}^{*}-\bar{c}_{S}\right)^{2}}{n}+\frac{(1+\gamma) \sum_{i=1}^{m}\left(\bar{c}_{S}-c_{i}\right)^{2}}{4}, \\
\pi_{j}^{*} & =\frac{[n(1+\gamma)-\gamma]\left(p_{j}^{*}-c_{j}\right)^{2}}{n}, \text { for each } j \in N \backslash S
\end{aligned}
$$

The multiproduct firm's average price is equal to $\bar{p}_{S}^{*}=\left(\sum_{i \in S} p_{i}^{*}\right) / m=$ $\frac{n(2 n(1+\gamma)-\gamma) V}{\omega_{2}}+\frac{(2 n+(m+n-1) \gamma)(n+(n-m) \gamma) \bar{c}_{S}}{\omega_{2}}+\frac{\gamma(n(1+\gamma)-\gamma)(n-m) \bar{c}_{-S}}{\omega_{2}}$,

and the equilibrium markups are: for each $j \in N \backslash S$ and each unit $i \in S$, 


$$
\begin{aligned}
p_{j}^{*}-c_{j} & =\frac{n q_{j}^{*}}{n(1+\gamma)-\gamma}, \\
p_{i}^{*}-c_{i} & =\frac{n(2 n(1+\gamma)-\gamma) V}{\omega_{2}}+\frac{\gamma(n(1+\gamma)-\gamma)(n-m) \bar{c}_{-S}}{\omega_{2}}+\frac{\gamma^{2} m(n-m) \bar{c}_{S}}{2 \omega_{2}}-\frac{c_{i}}{2} \\
\bar{p}_{S}^{*}-\bar{c}_{S} & =\frac{n(2 n(1+\gamma)-\gamma) V}{\omega_{2}}+\frac{\gamma(n(1+\gamma)-\gamma)(n-m) \bar{c}_{-S}}{\omega_{2}}-\frac{\omega_{3} \bar{c}_{S}}{\omega_{2}}
\end{aligned}
$$

where $\omega_{2}$ is given in $(25), q_{j}^{*}$ is given in $(26)$, and $\omega_{3}>0$ is given by

$$
\omega_{3}(n, m, \gamma)=\gamma^{2}(n-1)(n-m)+\gamma n(3 n-m-1)+2 n^{2} .
$$

Proposition 2 below reports the effects of small cost reductions in Bertrand oligopolies, whose closed-form expressions are given in (42-46) in Appendix A.

Proposition 2 Consider the Bertrand oligopoly (1) with a single multiproduct firm given by $\Delta=\{S, m+1, \ldots, n\}$.

(i) For each single-product firm $j \in N \backslash S$, a small reduction in $c_{j}$ increases its output and profit, and decreases all other outputs and all other firms' profits.

(ii) For each unit $i \in S$, a small reduction in $c_{i}$ increases output $i$, decreases all other outputs and all single-product firms' profits; and increases the multiproduct firm's profits if and only if its output share is above a critical level, or precisely if and only if $t_{i}^{S}>\widehat{t^{S}}=\gamma^{2}(n-m) / \omega_{2}$, where $t_{i}^{S}=q_{i}^{*} / \sum_{j=1}^{m} q_{j}^{*}$ is its output share among the products of $S$ and $\omega_{2}$ is given in (25).

An examination of the markups in (29) shows that a reduction in $c_{i}$ increases unit $i$ 's markup and decreases the markups in all other units (see (43) in the proof). Because unit $i$ 's output increases and the outputs in all other units decrease, a reduction in $c_{i}$ increases unit $i$ 's profits, but at the same time decreases the profits of all other units. The balance of these two opposite effects explains why a multiproduct firm might be unwilling to reduce its cost: small cost reductions in one product will reduce 
the multiproduct firm's profits if and only if the output share of the cost-reducing unit is below the critical level $\widehat{t}^{S}{ }^{4}$ Call a unit an efficient unit if its marginal cost is below the multiproduct firm's average marginal cost (i.e., $\bar{c}_{S}-c_{i}>0$ ). Then, as shown in the corollary below, cost reductions in an efficient unit will always increase the multiproduct firm's profits.

Corollary 1 (i) For each $i \in S, \partial \pi_{S}^{*} / \partial c_{i}<0$ if $c_{i}<\bar{c}_{S}$.

(ii) Let $m=n$ (i.e., $S=N$ ) and $\pi_{S}^{*}=\pi_{N}^{*}$ be the monopoly profit. Then, for all $i \in N, \partial \pi_{N}^{*} / \partial c_{i}<0$.

The negative profit effects of a small cost reduction also can be characterized by critical levels of marginal costs for each cost-reducing unit, which is given below:

Corollary 2 Given $\Delta=\{S, m+1, \ldots, n\}$, let $\pi_{S}^{*}$ be the multiproduct firm's profits. Then, for each $i \in S, \partial \pi_{S}^{*} / \partial c_{i}>0 \Leftrightarrow c_{i}>\widehat{c}_{i}^{S}$, where $\widehat{c}_{i}^{S}$ is the critical level of unit $i$ 's marginal cost given in (49) in Appendix A.

Corollary 2 can be understood geometrically as shown below. An examination of the profits in (27) indicates that $\pi_{S}^{*}=\pi_{S}^{*}\left(c_{i}\right)$ is convex and quadratic in $c_{i}$, with $\widehat{c}_{i}^{S}$ as its minimum point defined by $\partial \pi_{S}^{*} / \partial c_{i}=0$. Because $\pi_{S}^{*}$ is symmetric in $c_{i}$ around $c_{i}=\widehat{c}_{i}^{S}$, small reductions in $c_{i}$ reduce $\pi_{S}^{*}$ if and only if $c_{i}$ is on the right half of the profit curve where $\pi_{S}^{*}$ is increasing in $c_{i}$ (i.e., $c_{i}>\widehat{c}_{i}^{S}$ ).

The counterintuitive negative relationship between cost reduction in small units and the multiproduct firm's profits is caused by the combined strength of at least three forces. The first force is strategic complementarity in price competition (see Appendix B or Bulow et al. [1985] for definition). Without strategic complementarity, as in a

\footnotetext{
${ }^{4}$ Obviously, if a small reduction in unit $i$ 's marginal cost $c_{i}$ decreases the firm's profits, then a small reduction in the marginal cost of any other unit whose marginal cost is greater than $c_{i}$ will also reduce the firm's profits.
} 
Cournot model in which firms' choices are strategic substitutes, the profit effects of cost reductions will always be positive (see next section). The second force is strategic interaction between the multiproduct and single-product firms. Similar to Zhao and Howe (2004), their reaction functions (in terms of average prices, $\bar{p}_{S}=\sum_{i \in S} \bar{p}_{i} / m$ and $\left.\bar{p}_{-S}=\sum_{j \notin S} \bar{p}_{j} /(n-m)\right)$ are:

$$
\begin{gathered}
\bar{p}_{S}=h\left(\bar{p}_{-S}\right)=\frac{n V+(n+(n-m) \gamma) \bar{c}_{S}+\gamma(n-m) \bar{p}_{-S}}{2 n+(2 n-2 m) \gamma}, \text { and } \\
\bar{p}_{-S}=g\left(\bar{p}_{S}\right)=\frac{n V+(n+(n-1) \gamma) \bar{c}_{-S}+\gamma m \bar{p}_{S}}{2 n+(m+n-1) \gamma} .
\end{gathered}
$$

By (31), the multiproduct firm's cost reduction (i.e., a decrease in $\bar{c}_{S}$ ) directly causes a reduction in its average price. Such a reduction in $\bar{p}_{S}$, by (32), leads to a decrease in $\bar{p}_{-S}$, which causes a second-round reduction in $\bar{p}_{S}$ through the reaction curve (31). Without such strategic interactions, as in the monopoly case of Corollary 1, cost reductions will always increase the firm's profits.

Finally, the third force is the multiplicity of products, which allows internal reallocation of resources within a firm. As inputs are transferred from efficient and large units to the cost-reducing unit, outputs of all efficient units decrease, which causes the firm's overall profits to decrease.

As shown in Lemma 1 in Appendix B, small cost reductions could not reduce profit in homogeneous Cournot oligopolies even with strong strategic complementarity. This indicates that the above negative profit effect of small cost reductions is a unique feature of multiproduct Bertrand oligopolies. Although the assumptions of Lemma 1 are weaker than the standard assumptions in most previous studies, some readers still might believe that profit-reducing cost reductions could exist in homogeneous Cournot oligopolies after removing the assumptions of Lemma 1. We encourage such 
believers to find a counterexample. ${ }^{5}$

Observe that the negative profit effects are caused by small cost reductions in an inefficient unit. If the magnitude of cost reduction is sufficiently large, the profit effects will be positive. Proposition 3 below provides the critical magnitude above which large cost reductions will increase profits.

Proposition 3 Given $\Delta=\{S, m+1, \ldots, n\}$ in the Bertrand oligopoly (1), let unit 1 be the most efficient unit of $S$ (i.e., $c_{1}=\min \left\{c_{i} \mid 1 \leq i \leq m\right\}$ ). Consider each unit $i \in S$ with $c_{i}>\widehat{c}_{i}^{S}$, where $\widehat{c}_{i}^{S}$ is given in (49).

(i) A large reduction in $c_{i}$ increases the multiproduct firm's profits if and only if the reduction is larger than twice the difference between $c_{i}$ and $\widehat{c}_{i}^{S}$.

(ii) The multiproduct firm's profits will increase if $c_{i}$ is reduced to the most efficient level $c_{1}$.

Parts (i) and (ii) together imply that the magnitude of reducing $c_{i}$ to $c_{1}$ is larger than twice the difference between $c_{i}$ and $\widehat{c}_{i}^{S}$ (i.e., $\left.c_{i}-c_{1}>2\left(c_{i}-\widehat{c}_{i}^{S}\right)\right)$. In particular, it implies that a technology spillover within $S$ that reduces all units' marginal costs to $c_{1}$ will increase the multiproduct firm's profits. As shown in the proof, by the time unit $i$ becomes an efficient unit (i.e., its output share reaches $1 / m$, or equivalently, its marginal cost is reduced to the firm's average marginal cost), the multiproduct firm's profits would have risen above the initial level. When unit $i$ eventually becomes the

\footnotetext{
${ }^{5}$ One should be aware of at least three hurdles before embarking on such an enquiry. First, the existence of Cournot equilibrium with strategic complementarity is less known, because the known existence condition is that goods are strategic substitutes (i.e., $\alpha_{i}=p^{\prime}(X)+x_{i} p^{\prime \prime}(X) \leq 0$, see Novshek [1985] and Shapiro [1989]). Second, as Vives (2005) pointed out, strategic complementarity leads to equilibrium existence without the second-order conditions, which would require solving anti-decision problems such as maximizing a convex function on an interval $[0, y]$. Although such problems are relevant in game situations, it is hard to apply them in industrial organization because a firm's optimal choice will be either to shut down or to operate at full capacity (i.e., it can never be interior), which will destroy much of the existing wage theory and factor pricing theory. Third, the proof of Lemma 1 shows that $\partial \pi_{i}^{*} / \partial c_{i}<0$ holds if $E<-(n+1)$ (by $\alpha_{i}>0$ and by (57)). Hence, $\partial \pi_{i}^{*} / \partial c_{i}>0$ can possibly hold only in the tiny interval $[-n-1,-n-1 / 2]$ among all $E \in(-\infty, \infty)$, where $E=X p^{\prime \prime}(X) / p^{\prime}(X)$ is the elasticity of the slope of inverse demand.
} 
most efficient unit (i.e., its marginal cost reaches $c_{1}$ ), the multiproduct firm's profits will rise further.

Example 1 below illustrates the above results.

Example 1: Let $n=3, V=9, \gamma=2, c_{1}=5.9, c_{2}=7.23, c_{3}=4$, and $S=\{1,2\}$. One gets: $p_{1}^{*} \approx 6.8459, p_{2}^{*} \approx 7.5109, p_{3}^{*} \approx 5.9795 ; q_{1}^{*} \approx 2.0198, q_{2}^{*} \approx 0.0248$, $q_{3}^{*} \approx 4.6189$; and $\omega_{2}=132$. Consider $i=2$. By Proposition 2 and $t_{2}^{S}=$ $q_{2}^{*} /\left(q_{1}^{*}+q_{2}^{*}\right) \approx 0.0122<\widehat{t}^{S} \approx 0.0303$, or by Corollary 2 and $c_{2}=7.23>$ $\widehat{c}_{2}^{S} \approx 7.1968, \partial \pi_{S}^{*} / \partial c_{2}>0$ holds. Indeed, one can check that $\partial \pi_{S}^{*} / \partial c_{2} \approx$ $0.0371>0$. For a small reduction in $c_{2}$ from 7.23 to 7.2 , the multiproduct firm's profits will decrease from $\pi_{S}^{*}=\pi_{1}^{*}+\pi_{2}^{*} \approx 1.9176$ to $\tilde{\pi}_{S} \approx 1.9170$. However, a large reduction in $c_{2}$ from 7.23 to 7.15 will, by Proposition 3 and $\Delta c_{2}=0.08$ $>2\left(c_{i}-\widehat{c}_{i}^{S}\right) \approx 0.0664$, increase the profits from 1.9176 to $\tilde{\pi}_{S} \approx 1.9182$.

It is striking to see that a small increase in $c_{2}$ will raise $\pi_{S}^{*}$. For example, let $c_{2}$ be increased from 7.23 to $c_{2}^{*}=7.2518$, the profits will be raised to $\tilde{\pi}_{S}^{* *} \approx 1.9187>$ $\pi_{S}^{*} \approx 1.9176$, and $\widetilde{\pi}_{3}^{* *} \approx 9.1587>\pi_{3}^{*} \approx 9.1431$, with the new equilibrium outputs as: $\widetilde{q}_{1}^{* *} \approx 2.0277, \widetilde{q}_{2}^{* *} \approx 0.0000, \widetilde{q}_{3}^{* *} \approx 4.6228$. It is useful to note that $c_{2}$ has been raised to its upper bound $c_{2}^{*}=7.2518$, at which demand for the second product is zero.

The above analysis of a multiproduct firm's behavior implies a long list of interesting topics for future study. Below we discuss two such future topics. First, we do not wish to jump to an explanation why some firm's costs are high (we only explained why some firms are unwilling to reduce costs), although our numerical example indicates so (i.e., a firm could increase its profits by overly increasing its costs). ${ }^{6}$ However, we believe that our analysis can be modified so that future studies can explain why

\footnotetext{
${ }^{6}$ A real firm might not want to take such cost-increasing measures (such as paying its workers a higher wage), because the profit increase is small as compared with possible damage to its reputation, and the firm might be prevented from paying a higher wage to its workers in the small and inefficient unit by union contract, because workers in other (efficient) units also are entitled to a pay raise.
} 
some firms' costs are high, by analyzing, for example, a two-stage cost-setting model in which firms first choose costs and then engage in price competition.

Second, the above situation with a zero demand for an inefficient product provides a new approach to understanding multiproduct choices such as closing the production of an inefficient product, keeping some empty first-class seats by an airline, and exhibiting an astronomically priced item in a showroom that no one will buy. Recall that a single-product firm whose sales are zero is indifferent between exiting and staying. In the above situation with $\widetilde{q}_{2}^{* *}=0$, is the multiproduct firm also indifferent between keeping and closing this unit? The answer is no. Direct calculations show that if unit 2 is removed (i.e., $\mathrm{S}$ becomes a single-product firm), the new profits are: $\pi_{1}^{D} \approx 1.4603<\widetilde{\pi}_{S}^{C} \approx 2.1533, \pi_{3}^{D} \approx 7.6163<\widetilde{\pi}_{3}^{C} \approx 9.3677$, so both firm $S=\{1,2\}$ and firm 3 are worse off with the closing of the idled second unit. Proposition 4 below shows that this property holds in a large class of multiproduct oligopolies.

Proposition 4 Consider the Bertrand-Shubik oligopoly (1) with a single two-product firm given by $\Delta=\{S, 3, \ldots, n\}$, where $S=\{1,2\}$. Suppose that $c_{1}=c-\mu$ and $c_{i}=c$ for $i=2, \ldots, n$. Then there exists a unique $\mu>0$ such that the inferior inside firm 2 produces zero output in equilibrium. However, removing product 2 from the two-product firm will decrease all firms' profits.

Note that Proposition 4 shares a feature of the dominant cartel model in that the multiproduct firm's inefficient unit and all single-product firms have the same marginal costs. In such oligopolies, both the multi- and single-product firms are worse off if the high cost unit with a zero demand is closed by the multiproduct firm. Although this conclusion is derived from a simple model, its proof is quite involved due to the complexity of the problem. It remains to be seen if the conclusion can be extended to more general models. 


\section{Cost Reductions in Quantity Competition}

This section shows that the perverse profit effect of small cost reductions does not exist in quantity competition and that a multiproduct firm in quantity competition also has no incentive to close an inefficient unit whose demand is zero.

Substituting the Cournot equilibrium in (19-20) into the inverse demand (10) and simplifying, one obtains the equilibrium prices as given below: for each unit $i \in S$ and each single-product firm $j \in N \backslash S$,

$$
\begin{gathered}
p_{i}^{C *}=\frac{(2 n+\gamma)(n+m \gamma) V}{\omega_{1}}-\frac{m(n-m) \gamma^{2} \bar{c}_{S}}{2 \omega_{1}}+\frac{(n-m)(n+m \gamma) \gamma \bar{c}_{-S}}{\omega_{1}}+\frac{c_{i}}{2} \\
p_{j}^{C *}=\frac{(n+\gamma) q_{j}^{C *}}{n(1+\gamma)}+c_{j}
\end{gathered}
$$

which lead to the equilibrium profits as given below:

$$
\begin{aligned}
\pi_{S}^{C *} & =\frac{m n(1+\gamma)\left(\bar{p}_{S}^{C *}-\bar{c}_{S}\right)^{2}}{n+m \gamma}+\frac{(1+\gamma) \sum_{i=1}^{m}\left(\bar{c}_{S}-c_{i}\right)^{2}}{4} ; \text { and } \\
\pi_{j}^{C *} & =\frac{n(1+\gamma)\left(\bar{p}_{j}^{C *}-c_{j}\right)^{2}}{(n+\gamma)}, \text { all } j \in N \backslash S,
\end{aligned}
$$

where the multiproduct firm's average price is equal to $\bar{p}_{S}^{C *}=\left(\sum_{i \in S} p_{i}^{C *}\right) / m=$

$$
\frac{(2 n+\gamma)(n+m \gamma) V}{\omega_{1}}+\frac{\left(2 n^{2}+n(n+m+1) \gamma+m \gamma^{2}\right) \bar{c}_{S}}{\omega_{1}}+\frac{(n-m)(n+m \gamma) \gamma \bar{c}_{-S}}{\omega_{1}}
$$

and the equilibrium markups are: for each $j \in N \backslash S$ and each unit $i \in S$,

$$
\begin{aligned}
p_{j}^{C *}-c_{j} & =\frac{(n+\gamma) q_{j}^{C *}}{n(1+\gamma)} \\
p_{i}^{C *}-c_{i} & =\frac{(2 n+\gamma)(n+m \gamma) V}{\omega_{1}}-\frac{m(n-m) \gamma^{2} \bar{c}_{S}}{2 \omega_{1}}+\frac{(n-m)(n+m \gamma) \gamma \bar{c}_{-S}}{\omega_{1}}-\frac{c_{i}}{2}
\end{aligned}
$$


where $q_{j}^{C *}$ is given in $(20)$.

Direct calculations lead to the following effects of a firm's small cost reduction, whose closed-form expressions are given in (52-54) in Appendix A:

Proposition 5 Consider the Cournot oligopoly (10) with a single multiproduct firm.

(i) A small reduction in a single product firm's marginal cost increases its product and profit, and it reduces all other firms' products and profits.

(ii) A small reduction in the multiproduct firm's marginal cost $c_{i}$ increases its profit and product $i$, and it reduces all other products and all single-product firms' profits.

By the proposition, a Cournot firm's profits always increase after its cost reductions. Although such effects on multiproduct firm's profits are similar to those in single-product Cournot models, they are not as obvious as in single-product models. An examination of the markups in (37) indicates that (see (53) in proof) a reduction in unit $i$ 's marginal cost increases the markups in all of the multiproduct firm's products. Because the cost reduction increases product $i$ and decreases all other products, unit $i$ 's profit increases while profits in the multiproduct firm's other units might increase or decrease; such reasoning, therefore, leads to an ambiguous profit effects. Proposition 5 clarifies such ambiguity and shows that the overall effects on the multiproduct firm's profits are positive. The following numerical example illustrates such effects.

Example 2: Let $n=3, V=9, \gamma=2, c_{1}=5.9, c_{2}=7.2, c_{3}=4$, and $S=\{1,2\}$. One gets: $q_{1}^{C *} \approx 1.9636, q_{2}^{C *} \approx 0.0136, q_{3}^{C *} \approx 4.1045 ; \pi_{1}^{C *} \approx 2.1481, \pi_{2}^{C *} \approx 0.0061$, $\pi_{3}^{C *} \approx 9.3596$. By Proposition $5, \partial \pi_{S}^{C *} / \partial c_{i}<0(i=1,2)$ holds. Indeed, one can check that $\partial \pi_{S}^{C *} / \partial c_{1} \approx-2.0236<0$ and $\partial \pi_{S}^{C *} / \partial c_{2} \approx-0.0736<0$, so a small increase in either $c_{1}$ or $c_{2}$ will lower $\pi_{S}^{C *}$. For example, let $c_{2}$ be increased to $c_{2}=7.2125$. The new equilibrium becomes: $\widetilde{q}_{1}^{C} \approx 1.9687, \widetilde{q}_{2}^{C} \approx 0, \widetilde{q}_{3}^{C} \approx 4.1063$; 
and the new profits are: $\tilde{\pi}_{S}^{C} \approx \widetilde{\pi}_{1}^{C} \approx 2.1533<\pi_{S}^{C *}=\pi_{1}^{C *}+\pi_{2}^{C *} \approx 2.1542$, $\tilde{\pi}_{3}^{C} \approx 9.3677>\pi_{3}^{C *} \approx 9.3596$. Note that $c_{2}$ has been increased to its upper bound at which the demand for second product is zero.

Similar to price competition in Proposition 4, Proposition 6 below shows that a multiproduct firm in quantity competition also has no incentive to eliminate a product whose sales are zero.

Proposition 6 Consider the Cournot oligopoly (10) with a single two-product firm given by $\Delta=\{S, 3, \ldots, n\}$, where $S=\{1,2\}$. Suppose that $c_{1}=c-\mu$ and $c_{i}=c$ for $i=2, \ldots, n$. Then there exists a unique $\mu>0$ such that the inferior inside firm 2 produces zero output in equilibrium. However, removing product 2 from the two-product firm will decrease all firms' profits.

\section{Conclusion and Discussion}

We have provided closed-form expressions for both Bertrand and Cournot equilibria in multiproduct oligopolies with differentiated goods and asymmetric costs. Analyzing these expressions allowed us to provide two new understandings about a multiproduct firm's behavior.

First, we have shown that reducing a multiproduct firm's cost will reduce its profits in price competition if the cost-reducing unit is sufficiently small. This not only explains why firms are sometimes unwilling to reduce their cost, but also lends support to empirical observations that multiproduct firms do not always strive to improve the efficiency level of all units. For example, it is well documented that different units (and plants) within an auto manufacturer can have very different levels of efficiency, and that the variance in efficiency within a manufacturer can sometimes be greater than that between different manufacturers. More specifically, we have 
characterized the critical level of output share below which a small reduction in the marginal cost of a small unit reduces the multiproduct firm's profits, as well as the critical size of cost reduction above which a large reduction in the marginal cost of a small unit increases the multiproduct firm's profit.

Second, we have shown that a multiproduct firm has no incentive to eliminate an inefficient product with zero demand in both price and quantity competition, which also sheds lights on understanding other multiproduct choices, such as why airlines often keep some empty first-class seats and why suppliers often exhibit astronomically priced items in their showroom that no one will buy.

These two new results are obviously beyond the boundaries of single-product oligopoly studies, and they indicate that much more remains to be explored in understanding the behavior of multiproduct oligopolies. We hope readers will be encouraged to apply our expressions for a general multiproduct equilibrium in extending oligopoly studies from single- to multiproduct. Such extensions, we believe, are not only a significant step closer to reality, but also will be as rewarding as the extension of calculus from single to multi-variable.

\section{Appendix A}

The Inverse of $\mathbf{A}$ in (8) (see [19]): Let $U=\left(u_{i j}\right)_{n \times n}=A^{-1}$ denote the inverse of $\mathrm{A}$ given in (8). For $i=1, \ldots, k$, define

$$
\begin{gathered}
\beta_{i}=\left(n_{i}+\frac{a+\left(1-n_{i}\right) b}{c}\right)^{-1}=\frac{c}{a+b+(c-b) n_{i}}, \\
\alpha=\sum_{i=1}^{k} \beta_{i} n_{i}=c \sum_{i=1}^{k} \frac{n_{i}}{a+b+(c-b) n_{i}}
\end{gathered}
$$




$$
\theta_{i}=\frac{1}{1-\alpha}\left(\beta_{i} n_{i}+\frac{c}{b} \sum_{\substack{j=1 \\ j \neq i}}^{k} \beta_{j} n_{j}\right)=\frac{1}{1-\alpha}\left(\beta_{i} n_{i}+\frac{c}{b}\left(\alpha-\beta_{i} n_{i}\right)\right) \text {, and }
$$

assume $\alpha \neq 1$ and $a+b+(c-b) n_{i} \neq 0$, all $i$. Then, $A^{-1}=U=$

$$
\frac{1}{a+b} I+\frac{1}{c}\left(\begin{array}{cccc}
\frac{b \beta_{1}\left(1+\theta_{1}\right)}{(a+b)} E_{n_{1} \times n_{1}} & \frac{\beta_{1} \beta_{2}}{(1-\alpha)} E_{n_{1} \times n_{2}} & \cdots & \frac{\beta_{1} \beta_{k}}{(1-\alpha)} E_{n_{1} \times n_{k}} \\
\frac{\beta_{2} \beta_{1}}{(1-\alpha)} E_{n_{2} \times n_{1}} & \frac{b \beta_{2}\left(1+\theta_{2}\right)}{(a+b)} E_{n_{2} \times n_{2}} & \cdots & \frac{\beta_{2} \beta_{k}}{(1-\alpha)} E_{n_{2} \times n_{k}} \\
\vdots & \vdots & \ddots & \vdots \\
\frac{\beta_{k} \beta_{1}}{(1-\alpha)} E_{n_{k} \times n_{1}} & \frac{\beta_{k} \beta_{2}}{(1-\alpha)} E_{n_{k} \times n_{2}} & \cdots & \frac{b \beta_{k}\left(1+\theta_{k}\right)}{(a+b)} E_{n_{k} \times n_{k}}
\end{array}\right),
$$

where $E_{n_{i} \times n_{j}}$ is an $n_{i} \times n_{j}$ matrix of 1 s. In other words, the inverse $A^{-1}$, given below, has the same block structure as $A$ :

$$
A^{-1}=U=\left(\begin{array}{cccc}
U_{11} & U_{12} & \cdots & U_{1 k} \\
U_{21} & U_{22} & \cdots & U_{2 k} \\
\vdots & \vdots & \ddots & \vdots \\
U_{k 1} & U_{k 2} & \cdots & U_{k k}
\end{array}\right)
$$

where

$$
\begin{aligned}
U_{i i} & =\frac{1}{a+b} I_{n_{i} \times n_{i}}+\frac{b \beta_{i}\left(1+\theta_{i}\right)}{c(a+b)} E_{n_{i} \times n_{i}}, i=1, \ldots, k ; \text { and } \\
U_{i j} & =\frac{\beta_{i}\left(c+b \theta_{j}\right)}{c(a+b)} E_{n_{i} \times n_{j}}, \text { all } j \neq i .
\end{aligned}
$$

Q.E.D.

Proof of Proposition 1: Part (i). It follows from (13) and (39). 
Part (ii). The first order conditions in (14) can be rearranged as

$$
\begin{gathered}
{\left[\begin{array}{ccccccc}
2(n+\gamma) & 2 \gamma & \cdots & 2 \gamma & \gamma & \cdots & \gamma \\
2 \gamma & 2(n+\gamma) & \cdots & 2 \gamma & \gamma & \cdots & \gamma \\
\vdots & \vdots & \ddots & \vdots & \vdots & \vdots & \vdots \\
2 \gamma & 2 \gamma & \cdots & 2(n+\gamma) & \gamma & \cdots & \gamma \\
\gamma & \gamma & \cdots & \gamma & 2(n+\gamma) & \cdots & \gamma \\
\vdots & \vdots & \vdots & \vdots & \vdots & \ddots & \vdots \\
\gamma & \gamma & \cdots & \gamma & \gamma & \cdots & 2(n+\gamma)
\end{array}\right]\left(\begin{array}{c}
q_{1} \\
q_{2} \\
\vdots \\
q_{m} \\
q_{m+1} \\
\vdots \\
q_{n}
\end{array}\right)} \\
=\left(\begin{array}{c}
n(1+\gamma)\left(V-c_{1}\right) \\
n(1+\gamma)\left(V-c_{2}\right) \\
\vdots \\
n(1+\gamma)\left(V-c_{m}\right) \\
n(1+\gamma)\left(V-c_{m+1}\right) \\
\vdots \\
n(1+\gamma)\left(V-c_{n}\right)
\end{array}\right), \text { or } B q=\bar{d} .
\end{gathered}
$$

Applying the main inverse in (39) to (41) gives the following inverse for $B$ :

$$
B^{-1}=\left[\begin{array}{cc}
\frac{1}{2 n} I_{m} & 0 \\
0 & \frac{1}{2 n+\gamma} I_{n-m}
\end{array}\right]-\frac{\gamma}{\omega_{1}}\left[\begin{array}{cc}
\frac{4 n+(n-m+2) \gamma}{2 n} E_{m \times m} & E_{m \times(n-m)} \\
E_{(n-m) \times m} & \frac{2 n+m \gamma}{2 n+\gamma} E_{(n-m) \times(n-m)}
\end{array}\right],
$$

where $\omega_{1}>0$ is given by (21), $I_{k}$ is the $k \times k$ identity matrix and $E_{k \times j}$ is the $k \times j$ matrix of all 1s. The equilibrium is given by

$$
q^{C *}=B^{-1}\left(\begin{array}{c}
n(1+\gamma)\left(V-c_{1}\right) \\
\vdots \\
n(1+\gamma)\left(V-c_{n}\right)
\end{array}\right)=n(1+\gamma) B^{-1}\left(\begin{array}{c}
V-c_{1} \\
\vdots \\
V-c_{n}
\end{array}\right) .
$$

For each $i=1, \ldots, m$ (i.e., $i \in S$ ),

$$
q_{i}^{C *}=n(1+\gamma)\left[\frac{V-c_{i}}{2 n}-\frac{(4 n+(n-m+2) \gamma) m \gamma\left(V-\bar{c}_{S}\right)}{2 n \omega_{1}}-\frac{(n-m) \gamma\left(V-\bar{c}_{-S}\right)}{\omega_{1}}\right]
$$




$$
\begin{aligned}
= & \frac{n(1+\gamma)(2 n+\gamma) V}{\omega_{1}}+\frac{(1+\gamma)[4 n+(n-m+2) \gamma] m \gamma \bar{c}_{S}}{2 \omega_{1}} \\
& +\frac{n(1+\gamma)(n-m) \gamma \bar{c}_{-S}}{\omega_{1}}-\frac{(1+\gamma) c_{i}}{2}
\end{aligned}
$$

This gives (19). For each $j=m+1, \ldots, n$ (i.e., $j \in N \backslash S$ ),

$$
\begin{aligned}
q_{j}^{C *}=n(1+\gamma)\left[\frac{V-c_{j}}{2 n+\gamma}-\frac{m \gamma\left(V-\bar{c}_{S}\right)}{\omega_{1}}-\frac{(2 n+m \gamma)(n-m) \gamma\left(V-\bar{c}_{-S}\right)}{(2 n+\gamma) \omega_{1}}\right] \\
=\frac{n(1+\gamma)(2 n+m \gamma) V}{\omega_{1}}+\frac{n(1+\gamma) m \gamma \bar{c}_{S}}{\omega_{1}} \\
\quad+\frac{n(n-m)(1+\gamma)(2 n+m \gamma) \gamma \bar{c}_{-S}}{(2 n+\gamma) \omega_{1}}-\frac{n(1+\gamma) c_{j}}{2 n+\gamma}
\end{aligned}
$$

This gives (20).

Q.E.D.

Proof of Proposition 2: Part (i) The effects of single-product firm's cost reductions are straightforward.

Part (ii) For each $i \in S$, the effects of its cost reduction on $j \notin S$ are straightforward, so we only need to show the effects on each $j \in S$. Differentiating (26) and (29) with respect to $c_{i}$ leads to

$$
\begin{gathered}
\text { i) } \frac{\partial q_{j}^{*}}{\partial c_{i}}= \begin{cases}\frac{1+\gamma}{2}-\frac{\omega_{4}}{2 n \omega_{2}}>0 & \text { if } j \neq i, \\
-\frac{\omega_{4}}{2 n \omega_{2}}<0 & \text { if } j=i,\end{cases} \\
\text { ii) } \frac{\partial\left(p_{j}^{*}-c_{j}\right)}{\partial c_{i}}= \begin{cases}\frac{\gamma^{2}(n-m)}{2 \omega_{2}}>0 & \text { if } j \neq i, \\
\frac{\gamma^{2}(n-m)}{2 \omega_{2}}-\frac{1}{2}<0 & \text { if } j=i ;\end{cases}
\end{gathered}
$$

where $\omega_{2}$ is given by $(25)$, and $\omega_{4}>0$ is given by

$$
\begin{aligned}
\omega_{4}(n, m, \gamma)= & \gamma^{3}(n-m)\left[2(n-1)^{2}+n(m-1)\right]+\gamma^{2} n[(2 n-m)(2 n+m-5) \\
& +n(4 n-3 m-1)+2]+2 \gamma n^{2}(5 n-m-3)+4 n^{3} .
\end{aligned}
$$


For $j \neq i$, the positive sign of $\partial q_{j}^{*} / \partial c_{i}$ follows from

$$
\frac{\partial q_{j}^{*}}{\partial c_{i}}=\frac{1+\gamma}{2}-\frac{\omega_{4}}{2 n \omega_{2}}=\frac{\left[\gamma^{2}(3 n-2)(n-m)+\gamma n(7 n-3 m-2)+4 n^{2}\right] \gamma}{2 n \omega_{2}}>0
$$

and the negative sign of $\partial\left(p_{i}^{*}-c_{i}\right) / \partial c_{i}$ follows from $\partial\left(p_{i}^{*}-c_{i}\right) / \partial c_{i}=$

$$
\frac{\gamma^{2}(n-m)}{2 \omega_{2}}-\frac{1}{2}=-\frac{4 n^{2}+2 n \gamma(3 n-m-1)+\gamma^{2}(n-m)(2 n+m-3)}{2 \omega_{2}}<0 .
$$

By (42) and (43), the effects on unit $i$ 's and $j$ 's output and markup satisfy the following properties: for $i \neq j \in S$,

$$
\begin{aligned}
\frac{\partial q_{i}^{*}}{\partial c_{i}} & =\frac{\partial q_{j}^{*}}{\partial c_{i}}-\frac{1+\gamma}{2}, \text { and } \\
\frac{\partial\left(p_{i}^{*}-c_{i}\right)}{\partial c_{i}} & =\frac{\partial\left(p_{j}^{*}-c_{j}\right)}{\partial c_{i}}-\frac{1}{2} .
\end{aligned}
$$

Using (42-43) and (45), one has

$$
\begin{gathered}
\frac{\partial \pi_{S}^{*}}{\partial c_{i}}=\frac{\partial \sum_{j=1}^{m} \pi_{j}^{*}}{\partial c_{i}}=\frac{\partial\left[\left(p_{i}^{*}-c_{i}\right) q_{i}^{*}\right]}{\partial c_{i}}+\sum_{j \in S \backslash i} \frac{\partial\left[\left(p_{j}^{*}-c_{j}\right) q_{j}^{*}\right]}{\partial c_{i}}= \\
-\frac{q_{i}^{*}+(1+\gamma)\left(p_{i}^{*}-c_{i}\right)}{2}+\frac{\partial\left(p_{k}^{*}-c_{k}\right)}{\partial c_{i}} \sum_{j \in S} q_{j}^{*}+\frac{\partial q_{k}^{*}}{\partial c_{i}} \sum_{j \in S}\left(p_{j}^{*}-c_{j}\right), \text { for any } k \neq i \in S, \\
=-\frac{q_{i}^{*}+(1+\gamma)\left(p_{i}^{*}-c_{i}\right)}{2}+\frac{\gamma^{2}(n-m) \sum_{j=1}^{m} q_{j}^{*}}{2 \omega_{2}} \\
+\frac{\left[\gamma^{2}(3 n-2)(n-m)+\gamma n(7 n-3 m-2)+4 n^{2}\right] \gamma \sum_{j=1}^{m}\left(p_{j}^{*}-c_{j}\right)}{2 n \omega_{2}} .
\end{gathered}
$$


Applying (4-5) with $n_{j}=m$ to the above expression, one has

$$
\begin{aligned}
\frac{\partial \pi_{S}^{*}}{\partial c_{i}}= & -\frac{2 n q_{i}^{*}+m \gamma\left(\bar{p}_{S}^{*}-\bar{c}_{S}\right)}{2 n}+\frac{\gamma^{2}(n-m) \sum_{j=1}^{m} q_{j}^{*}}{2 \omega_{2}} \\
& +\frac{\left[\gamma^{2}(3 n-2)(n-m)+\gamma n(7 n-3 m-2)+4 n^{2}\right] \gamma \sum_{j=1}^{m} q_{j}^{*}}{2(n(1+\gamma)-m \gamma) \omega_{2}} \\
= & -q_{i}^{*}-\frac{n \gamma \sum_{j=1}^{m} q_{j}^{*}}{2 n(n(1+\gamma)-m \gamma)}+\frac{\gamma^{2}(n-m) \sum_{j=1}^{m} q_{j}^{*}}{2 \omega_{2}} \\
& +\frac{\left[\gamma^{2}(3 n-2)(n-m)+\gamma n(7 n-3 m-2)+4 n^{2}\right] \gamma \sum_{j=1}^{m} q_{j}^{*}}{2(n(1+\gamma)-m \gamma) \omega_{2}} \\
= & -q_{i}^{*}+\frac{\gamma^{2}(n-m) \sum_{j=1}^{m} q_{j}^{*}}{\omega_{2}}
\end{aligned}
$$

which leads to

$$
\frac{\partial \pi_{S}^{*}}{\partial c_{i}}>0 \Leftrightarrow t_{i}^{S}<\widehat{t}^{S}=\frac{\gamma^{2}(n-m)}{\omega_{2}} .
$$

\section{Q.E.D.}

Proof of Corollary 1: (i) By (23), (26), (28), and (29),

$$
\left(p_{i}^{*}-c_{i}\right)-\left(\bar{p}_{S}^{*}-\bar{c}_{S}\right)=\frac{\gamma^{2} m(n-m)+2 \omega_{3}}{2 \omega_{2}} \bar{c}_{S}-\frac{c_{i}}{2}=\frac{\bar{c}_{S}-c_{i}}{2},
$$

which leads to $q_{i}^{*}-\bar{q}_{S}^{*}=$

$$
\frac{\left[\gamma^{2}(3 n-2)(n-m)+\gamma n(7 n-3 m-2)+4 n^{2}\right] m \gamma \bar{c}_{S}}{2 n \omega_{2}}-\frac{(1+\gamma) c_{i}}{2}+\frac{(n(1+\gamma)-m \gamma) \omega_{3} \bar{c}_{S}}{n \omega_{3}}
$$


$=(1+\gamma)\left(\bar{c}_{S}-c_{i}\right) / 2$, which in turn leads to

$$
\begin{aligned}
\left(p_{i}^{*}-c_{i}\right)-\left(\bar{p}_{S}^{*}-\bar{c}_{S}\right) & =\frac{\bar{c}_{S}-c_{i}}{2}, \text { and } \\
q_{i}^{*}-\bar{q}_{S}^{*} & =\frac{(1+\gamma)\left(\bar{c}_{S}-c_{i}\right)}{2} .
\end{aligned}
$$

Substituting (48) into the expression for $\partial \pi_{S}^{*} / \partial c_{i}$ in the proof of Proposition 2, one has:

$$
\frac{\partial \pi_{S}^{*}}{\partial c_{i}}=\frac{m \gamma^{2}(n-m) \bar{q}_{S}^{*}}{\omega_{2}}-\bar{q}_{S}^{*}-\frac{(1+\gamma)\left(\bar{c}_{S}-c_{i}\right)}{2}=\frac{-2 \omega_{3} \bar{q}_{S}^{*}}{\omega_{2}}-\frac{(1+\gamma)\left(\bar{c}_{S}-c_{i}\right)}{2} .
$$

By $\omega_{2}>0$ and $\omega_{3}>0, \bar{c}_{S}-c_{i}>0$ implies $\partial \pi_{S}^{*} / \partial c_{i}<0$, so part $(i)$ holds.

(ii) When $m=n, \widehat{t}^{S}=0$. By (46), $\partial \pi_{S}^{*} / \partial c_{i}>0$ is impossible, so $\partial \pi_{S}^{*} / \partial c_{i}<0$ holds for all $i$.

Q.E.D.

Proof of Corollary 2: By $(27), \pi_{S}^{*}=\pi_{S}^{*}\left(c_{i}\right)$ is convex and quadratic in $c_{i}$, and its minimum point $\widehat{c}_{i}^{S}$, or the solution of $\partial \pi_{S}^{*} / \partial c_{i}=0$, is given by

$$
\widehat{c}_{i}^{S}=\frac{\omega_{5}}{4(n(1+\gamma)-m \gamma)\left(\omega_{3}\right)^{2}+n(m-1)(1+\gamma)\left(\omega_{2}\right)^{2}},
$$

where $\omega_{2}$ and $\omega_{3}$ are given in (25) and (30), and $\omega_{5}>0$ is given by

$$
\begin{aligned}
\omega_{5}(n, m, \gamma)= & 4 m(n(1+\gamma)-m \gamma) \omega_{3}\left[n(2 n(1+\gamma)-\gamma) V+\gamma(n(1+\gamma)-\gamma)(n-m) \bar{c}_{-S}\right] \\
& +\left[n(1+\gamma)\left(\omega_{2}\right)^{2}-4(n(1+\gamma)-m \gamma)\left(\omega_{3}\right)^{2}\right] \Sigma_{j \in S \backslash i} c_{j} .
\end{aligned}
$$

Because $\pi_{S}^{*}$ is symmetric in $c_{i}$ around $c_{i}=\widehat{c}_{i}^{S}$, small reductions in $c_{i}$ reduce $\pi_{S}^{*} \Longleftrightarrow$ $c_{i}$ is on the right half of the profit curve where $\pi_{S}^{*}$ is increasing in $c_{i}$. Q.E.D.

Proof of Proposition 3: (i) Let $\delta_{i}=c_{i}-\widehat{c}_{i}^{S}>0$. One has $\pi_{S}^{*}\left(c_{i}-\delta_{i}\right)=\pi_{S}^{*}\left(\widehat{c}_{i}^{S}\right)=$ $\operatorname{Min}\left\{\pi_{S}^{*}\left(c_{i}\right) \mid c_{i} \geq 0\right\}$. By the symmetry of $\pi_{S}^{*}\left(c_{i}\right)$ around $\widehat{c}_{i}^{S}, \pi_{S}^{*}\left(c_{i}-2 \delta_{i}\right)=$ 
$\pi_{S}^{*}\left(c_{i}\right)$. Therefore, $\Delta c_{i}>2 \delta_{i}$ implies $\pi_{S}^{*}\left(c_{i}-\Delta c_{i}\right)>\pi_{S}^{*}\left(c_{i}-2 \delta_{i}\right)=\pi_{S}^{*}\left(c_{i}\right)$, which leads to (50). The reverse also holds obviously. Suppose the multiproduct firm's most efficient product is good 1 (i.e., $c_{1}=\min \left\{c_{i} \mid 1 \leq i \leq m\right\}$ ). For $i \in S$ with $c_{i}>\widehat{c}_{i}^{S}$, where $\widehat{c}_{i}^{S}$ is given in (49), let $\Delta c_{i}>0$ be the reduction in $c_{i}$. Let $\pi_{S}^{*}\left(c_{i}\right)$ denote the multiproduct firm's profits given in (27) when firm $i$ 's marginal cost is $c_{i}$. Then, the following two claims hold:

$$
\begin{gathered}
\text { (i) } \pi_{S}^{*}\left(c_{i}-\Delta c_{i}\right)>\pi_{S}^{*}\left(c_{i}\right) \Leftrightarrow \Delta c_{i}>2\left(c_{i}-\widehat{c}_{i}^{S}\right) ; \\
\text { (ii) } c_{i}-c_{1}>2\left(c_{i}-\widehat{c}_{i}^{S}\right) .
\end{gathered}
$$

(ii) By

$$
\begin{gathered}
\frac{\gamma^{2}(n-m)}{\omega_{2}}-\frac{1}{2 m+2}=\frac{\gamma^{2}(n-m)(2 m+2)-\omega_{2}}{(2 m+2) \omega_{2}} \\
=-\frac{\gamma^{2}(n-m)(2 n-m-4)+2 n \gamma(3 n-m-1)+4 n^{2}}{(2 m+2) \omega_{2}}<0,
\end{gathered}
$$

the critical output share $\widehat{t}^{S}$ in (46) satisfies

$$
\widehat{t}^{S}<\frac{1}{2 m+2} .
$$

As insider $i$ keeps reducing its marginal $\operatorname{cost}$ from $c_{i}>\widehat{c}_{i}^{S}$ to $\widehat{c}_{i}^{S}$ and eventually to $c_{1}$, its output share will increase from below $\widehat{t}^{S}$ to above $\widehat{t}^{S}$, further to $1 / m>\widehat{t}^{S}$, and eventually to above $1 / m$, because firm 1 is the most efficient insider. When its marginal cost falls below $\widehat{c}_{i}^{S}$, the multiproduct firm's profits will start to increase.

By (51) and by $t_{i}^{S}<\widehat{t}^{S}$,

$$
\widehat{t}^{S}-t_{i}^{S}<\frac{1}{2 m+2}<\frac{1}{2 m} .
$$

However, (51) also implies

$$
\frac{1}{m}-\widehat{t}^{S}>\frac{1}{m}-\frac{1}{2 m+2}=\frac{m+2}{(2 m+2) m}>\frac{1}{2 m} .
$$


Because insiders' outputs in (26) are linear in marginal costs, the above two inequalities imply that the reduction in $c_{i}$ equivalent to a share increase from $\widehat{t}^{S}$ to $1 / m$ is much larger than $\delta_{i}=c_{i}-\widehat{c}_{i}^{S}>0$, which is the reduction in $c_{i}$ equivalent to a share increase from $t_{i}^{S}$ to $\widehat{t}^{S}$. Because more reductions are needed for $c_{i}$ to eventually reach $c_{1}$ (i.e., for its output share to increase from $1 / m$ to firm 1 s output share in $S$ ), one must have $c_{i}-c_{1}>2 \delta_{i}$, which completes the proof of part (ii).

Q.E.D.

Proof of Proposition 4: Substituting $m=2, \omega_{2}=2 n\left[(n-2) \gamma^{2}+3(n-1) \gamma+2 n\right]$, $c_{1}=c-\mu$ and $c_{i}=c$ for $i>1$ into (26), one gets the following equilibrium outputs:

$$
\begin{aligned}
q_{1}^{*}= & \frac{[2 n(1+\gamma)-\gamma][n(1+\gamma)-2 \gamma](V-c)}{2 n\left[\gamma^{2}(n-2)+3 \gamma(n-1)+2 n\right]} \\
& +\frac{\gamma^{3}(n-2)\left(2 n^{2}-3 n+2\right)+\gamma^{2} n\left(8 n^{2}-17 n+8\right)+10 \gamma n^{2}(n-1)+4 n^{3}}{4 n^{2}\left[\gamma^{2}(n-2)+3 \gamma(n-1)+2 n\right]} \mu, \\
q_{2}^{*}= & \frac{[2 n(1+\gamma)-\gamma][n(1+\gamma)-2 \gamma](V-c)}{2 n\left[\gamma^{2}(n-2)+3 \gamma(n-1)+2 n\right]}-\frac{\gamma\left[\gamma^{2}(n-2)(3 n-2)+\gamma n(7 n-8)+4 n^{2}\right] \mu}{4 n^{2}\left[\gamma^{2}(n-2)+3 \gamma(n-1)+2 n\right]}, \\
q_{j}^{*}= & \frac{[n(1+\gamma)-\gamma]^{2}(V-c)}{n\left[\gamma^{2}(n-2)+3 \gamma(n-1)+2 n\right]}-\frac{\gamma[n(1+\gamma)-\gamma][n(1+\gamma)-2 \gamma] \mu}{4 n^{2}\left[\gamma^{2}(n-2)+3 \gamma(n-1)+2 n\right]}
\end{aligned}
$$

for $j=3, \ldots, n$. It is straightforward to verify that $q_{1}^{*}>q_{j}^{*}>q_{2}^{*}$. Based on the above expression for $q_{2}^{*}, q_{2}^{*}=0$ if

$$
\mu=\mu^{B} \equiv \frac{2 n[2 n(1+\gamma)-\gamma][n(1+\gamma)-2 \gamma]}{\gamma\left[\gamma^{2}(n-2)(3 n-2)+\gamma n(7 n-8)+4 n^{2}\right]}(V-c) .
$$

Given $\mu=\mu^{B}$, the equilibrium profits become:

$$
\pi_{S}^{*}=\left(p_{1}^{*}-c_{1}\right) q_{1}^{*} ; \pi_{j}^{*}=\frac{n}{n(1+\gamma)-\gamma}\left(q_{j}^{*}\right)^{2}, j=3, \ldots, n,
$$


where

$$
\begin{aligned}
q_{1}^{*}= & \left\{1+\frac{\left[\gamma^{2}(2 n-1)(n-2)+6 \gamma n(n-1)+4 n^{2}\right][n(1+\gamma)-2 \gamma]}{\gamma\left[\gamma^{2}(n-2)(3 n-2)+\gamma n(7 n-8)+4 n^{2}\right]}\right\} \\
& \times \frac{[2 n(1+\gamma)-\gamma][n(1+\gamma)-2 \gamma]}{2 n\left[\gamma^{2}(n-2)+3 \gamma(n-1)+2 n\right]}(V-c), \\
p_{1}^{*}-c_{1}= & \left\{1+\frac{\gamma^{3}(n-2)\left(2 n^{2}-3 n+2\right)+\gamma^{2} n\left(8 n^{2}-17 n+8\right)+10 \gamma n^{2}(n-1)+4 n^{3}}{\gamma\left[\gamma^{2}(n-2)(3 n-2)+\gamma n(7 n-8)+4 n^{2}\right]}\right\} \\
& \times \frac{[2 n(1+\gamma)-\gamma]}{2\left[\gamma^{2}(n-2)+3 \gamma(n-1)+2 n\right]}(V-c), \text { and for } j=3, \ldots, n, \\
& q_{j}^{*}=\frac{n(1+\gamma)[n(1+\gamma)-\gamma]}{\gamma^{2}(n-2)(3 n-2)+\gamma n(7 n-8)+4 n^{2}}(V-c) .
\end{aligned}
$$

If product 2 is removed, the industry becomes a single-product oligopoly with (n-1) firms. Evaluated at $c_{1}=c-\mu^{B}$ and $c_{j}=c$ for $j>1$, the new equilibrium profits are:

$$
\begin{aligned}
\pi_{1}^{0} & =\frac{(n-1)(1+\gamma)-\gamma}{n-1}\left(p_{1}^{0}-c_{1}\right)^{2}, \text { and } \\
\pi_{j}^{0} & =\frac{n-1}{(n-1)(1+\gamma)-\gamma}\left(q_{j}^{0}\right)^{2}, j=3, \ldots, n,
\end{aligned}
$$

where $p_{1}^{0}-c_{1}=$

$$
\begin{aligned}
& \frac{\phi(n, \gamma)(V-c)}{\gamma[(n-1)(2+\gamma)-\gamma][2(n-1)(1+\gamma)-\gamma]\left[\gamma^{2}(n-2)(3 n-2)+\gamma n(7 n-8)+4 n^{2}\right]}, \\
& q_{j}^{0}=\frac{1}{(n-1)(2+\gamma)-\gamma}(V-c)\{(n-1)- \\
& \\
& \left.\quad \frac{2 n[(n-1)(1+\gamma)-\gamma][2 n(1+\gamma)-\gamma][n(1+\gamma)-2 \gamma]}{[(n-1)(2+\gamma)-\gamma][2(n-1)(1+\gamma)-\gamma]\left[\gamma^{2}(n-2)(3 n-2)+\gamma n(7 n-8)+4 n^{2}\right]}\right\},
\end{aligned}
$$




$$
\begin{aligned}
\phi(n, \gamma)= & r^{4}(n-2)\left(11 n+5 n^{2}-12 n^{3}+4 n^{4}-6\right) \\
& +r^{3}\left(105 n^{3}-15 n^{2}-36 n-84 n^{4}+20 n^{5}+8\right) \\
& +4 r^{2} n\left(32 n^{2}-7 n-31 n^{3}+9 n^{4}-2\right) \\
& +4 r n^{2}(n-1)\left(7 n^{2}-12 n+3\right)+8 n^{3}(n-1)^{2} .
\end{aligned}
$$

Applying above expressions to $\left(\pi_{j}^{*}-\pi_{j}^{0}\right)$ and $\left(\pi_{S}^{*}-\pi_{1}^{0}\right)$, one gets: $\left(\pi_{j}^{*}-\pi_{j}^{0}\right)=$

$$
\begin{gathered}
\frac{h(n, \gamma)(V-c)^{2}}{(n-1)[(n-1)(2+\gamma)-\gamma]^{4}[2(n-1)(1+\gamma)-\gamma]^{2}\left[\gamma^{2}(n-2)(3 n-2)+\gamma n(7 n-8)+4 n^{2}\right]^{2}}, \\
\pi_{S}^{*}-\pi_{1}^{0}=\frac{k(n, \gamma)}{\varphi(n, \gamma)}(V-c)^{2},
\end{gathered}
$$

where the terms $h(n, \gamma), k(n, \gamma)$, and $\varphi(n, \gamma)$ are too long to be reported here. A complete proof with such details is given in Appendix C, which is available to readers upon request. These three polynomials share the feature that the highest order term has a positive coefficient. It is straightforward, although tedious, to show that these three terms are positive. To see $h(n, \gamma)>0$, one first shows $h(3, \gamma)>0$. Then, using Maple software, one can show that none of the real roots for $n$ in $h(n, \gamma)=0$ is greater than 2 , which implies that $h(n, \gamma)>0$ holds for all $n>2$. Similarly, one can show $k(n, \gamma)>0$ and $\varphi(n, \gamma)>0$. Hence, $\pi_{j}^{*}>\pi_{j}^{0}$ and $\pi_{S}^{*}>\pi_{1}^{0}$ hold.

Q.E.D.

Derivation for (33)-(37): Using (19-20), the industry's average output is

$$
\begin{aligned}
\bar{q}^{C *}= & \frac{1}{n}\left[\sum_{i \in S} q_{i}^{C *}+\sum_{i \notin S} q_{i}^{C *}\right] \\
= & \frac{(1+\gamma)\left(2 n^{2}+m(n-m+1) \gamma\right) V}{\omega_{1}}-\frac{m(1+\gamma)(2 n+\gamma) \bar{c}_{S}}{\omega_{1}} \\
& -\frac{(n-m)(1+\gamma)(2 n+m \gamma) \bar{c}_{-S}}{\omega_{1}} .
\end{aligned}
$$

Applying this expression and (19) to the inverse demand equation (10) gives (33-34). (35-37) are straightforward.

Q.E.D. 
Proof of Proposition 5: Part (i) For each $i \notin S$, differentiating (19-20) with respect to $c_{i}$ leads to

$$
\frac{\partial q_{j}^{C *}}{\partial c_{i}}=\left\{\begin{array}{cl}
\frac{n(1+\gamma) \gamma}{\omega_{1}}>0 & \text { if } j \in S ; \\
\frac{n(1+\gamma)(2 n+m \gamma) \gamma}{(2 n+\gamma) \omega_{1}}>0 & \text { if } j \notin S, j \neq i \\
\frac{-n(1+\gamma)\left[m(n+1-m) \gamma^{2}+2 n(n+m) \gamma+4 n^{2}\right]}{(2 n+\gamma) \omega_{1}}<0 & \text { if } j \notin S, j=i .
\end{array}\right.
$$

The profit effects follow from (35-37) and the above product effects.

Part (ii) For each $i \in S$, the effects of its cost reduction on a single-product firm $j \notin S$ are straightforward, so we only need to show the effects on each $j \in S$. Differentiating (19) and (37) with respect to $c_{i}$ leads to

$$
\begin{gathered}
\frac{\partial q_{j}^{C *}}{\partial c_{i}}= \begin{cases}\frac{\gamma(1+\gamma)(4 n+(n-m+2) \gamma)}{2 \omega_{1}}>0 & \text { if } j \neq i, \\
\frac{\gamma(1+\gamma)(4 n+(n-m+2) \gamma)}{2 \omega_{1}}-\frac{1+\gamma}{2}<0 & \text { if } j=i\end{cases} \\
\frac{\partial\left(p_{j}^{C *}-c_{j}\right)}{\partial c_{i}}= \begin{cases}-\frac{(n-m) \gamma^{2}}{2 \omega_{1}}<0 & \text { if } j \neq i, \\
-\frac{(n-m) \gamma^{2}}{2 \omega_{1}}-\frac{1}{2}<0 & \text { if } j=i .\end{cases}
\end{gathered}
$$

The negative sign of $\partial q_{i} / \partial c_{i}$ follows from

$$
\begin{aligned}
\frac{\partial q_{i}^{C *}}{\partial c_{i}} & =\frac{\gamma(1+\gamma)(4 n+(n-m+2) \gamma)}{2 \omega_{1}}-\frac{1+\gamma}{2} \\
& =-\frac{(1+\gamma)\left[4 n^{2}+2 n(n+m-1) \gamma+(m-1)(n-m+2) \gamma^{2}\right]}{2 \omega_{1}}<0
\end{aligned}
$$

Now, consider the profit effects. for any $i \neq j \in S$, (52-53) lead to

$$
\begin{aligned}
\frac{\partial q_{i}^{C *}}{\partial c_{i}} & =\frac{\partial q_{j}^{C *}}{\partial c_{i}}-\frac{1+\gamma}{2}, \text { and } \\
\frac{\partial\left(p_{i}^{C *}-c_{i}\right)}{\partial c_{i}} & =\frac{\partial\left(p_{j}^{C *}-c_{j}\right)}{\partial c_{i}}-\frac{1}{2} .
\end{aligned}
$$


Using (52-54), one has

$$
\begin{gathered}
\frac{\partial \pi_{S}^{C *}}{\partial c_{i}}=\frac{\partial \sum_{j=1}^{m} \pi_{j}^{C *}}{\partial c_{i}}=\frac{\partial \sum_{j=1}^{m}\left(p_{j}^{C *}-c_{j}\right) q_{j}^{C *}}{\partial c_{i}} \\
=\frac{q_{i}^{C *} \partial\left(p_{i}^{C *}-c_{i}\right)}{\partial c_{i}}+\frac{\left(p_{i}^{C *}-c_{i}\right) \partial q_{i}^{C *}}{\partial c_{i}}+\sum_{j \in S \backslash i}\left[\frac{q_{j}^{C *} \partial\left(p_{j}^{C *}-c_{j}\right)}{\partial c_{i}}+\frac{\left(p_{j}^{C *}-c_{j}\right) \partial q_{j}^{C *}}{\partial c_{j}}\right] \\
=-\frac{q_{i}^{C *}+(1+\gamma)\left(p_{i}^{C *}-c_{i}\right)}{2} \\
\quad+\sum_{j \in S}\left[-\frac{q_{j}^{C *} \gamma^{2}(n-m)}{2 \omega_{1}}+\frac{\left(p_{j}^{C *}-c_{j}\right) \gamma(1+\gamma)(4 n+(n-m+2) \gamma)}{2 \omega_{1}}\right] \\
+\frac{q_{i}^{C *}+(1+\gamma)\left(p_{i}^{C *}-c_{i}\right)}{2}-\frac{m(n-m) \gamma^{2} \bar{q}_{S}^{C *}}{2 \omega_{1}} \\
+\frac{m \gamma(1+\gamma n+(n-m+2) \gamma]\left(\bar{p}_{S}^{C *}-\bar{c}_{S}\right)}{2 \omega_{1}}
\end{gathered}
$$

Applying the relationship

$$
(1+\gamma)\left(p_{i}-c_{i}\right)=q_{i}+\frac{m \gamma \bar{q}_{S}}{n} \text { and } \frac{\bar{p}_{S}-\bar{c}_{S}}{\bar{q}_{S}}=\frac{n+m \gamma}{n(1+\gamma)}
$$

given in (15-16), one has

$$
\begin{aligned}
\frac{\partial \pi_{S}^{C *}}{\partial c_{i}}= & -\frac{q_{i}^{C *}+q_{i}^{C *}+\frac{m \gamma \bar{q}_{S}^{C *}}{n}-\frac{m \gamma^{2}(n-m) \bar{q}_{S}^{C *}}{2 \omega_{1}}}{2} \\
& +\frac{m \gamma(1+\gamma)(4 n+(n-m+2) \gamma)}{2 \omega_{1}} \frac{(n+m \gamma) \bar{q}_{S}^{C *}}{n(1+\gamma)} \\
= & -q_{i}^{C *}-\left[\frac{m \gamma}{2 n}+\frac{m \gamma^{2}(n-m)}{2 \omega_{1}}-\frac{m \gamma(4 n+(n-m+2) \gamma)(n+m \gamma)}{2 n \omega_{1}}\right] \bar{q}_{S}^{C *} \\
= & -q_{i}^{C *}-\frac{m(n-m) \gamma^{2}}{\omega_{1}} \bar{q}_{S}^{C *}<0
\end{aligned}
$$

Q.E.D. 
Proof of Proposition 6: The proof is similar to that of Proposition 4. A complete proof with detailed terms is given in Appendix $\mathrm{C}$, which is available upon request.

Q.E.D.

\section{Appendix B}

The profit functions in a homogeneous Cournot oligopoly with liner costs are $\pi_{i}(x)=$ $\left(p\left(\Sigma x_{j}\right)-c_{i}\right) x_{i}$, all $i$. Let $X=\Sigma x_{j}$, then firm $i$ 's and $j$ 's $(j \neq i)$ choices are called strategic substitutes if

$$
\alpha_{i}=\partial^{2} \pi_{i} / \partial x_{i} \partial x_{j}=p^{\prime}(X)+x_{i} p^{\prime \prime}(X) \leq 0,
$$

and strategic complements if $\alpha_{i}>0$. Let $E=X p^{\prime \prime}(X) / p^{\prime}(X)$ be the elasticity of the slope of inverse demand, $s_{i}=x_{i} / X$ be firm $i$ 's market share. By $\alpha_{i}>0 \Leftrightarrow$ $-\alpha_{i} / p^{\prime}(X)=-\left(1+s_{i} E\right)>0$, strategic complementarity is equivalent to

$$
-s_{i} E>1
$$

Dixit (1986) showed that the stability of the system (i.e., conditions for comparative statistics) requires

$$
\Delta=1+\Sigma\left(\alpha_{i} / p^{\prime}(X)\right)=n+1+E>0
$$

and most previous works on comparative statics have assumed a much stronger condition, $E>-1$ (see Shapiro [1989] for survey).

Lemma 1 Consider a firm $i$ in the above homogeneous Cournot oligopoly with nonlinear demands and linear costs. Let $\pi_{i}^{*}$ be its equilibrium profits, and assume: $\left.i\right)$ $\alpha_{i}>0$; and $\left.i i\right) E>-(n+1 / 2)$. Then, $\partial \pi_{i}^{*} / \partial c_{i}<0$ holds.

Proof of Lemma 1: Simplifying the output effects of a small cost reduction in Smythe and Zhao (2006, p. 184) leads to:

$$
\frac{\partial x_{i}}{\partial c_{i}}=\frac{(n+1+E) p^{\prime}(X)-\left[p^{\prime}(X)+x_{i} p^{\prime \prime}(X)\right]}{(n+1+E)\left(p^{\prime}(X)\right)^{2}},
$$




$$
\frac{\partial x_{k}}{\partial c_{i}}=\frac{-\left(p^{\prime}(X)+x_{k} p^{\prime \prime}(X)\right)}{(n+1+E)\left(p^{\prime}(X)\right)^{2}}, \text { all } k \neq i .
$$

Using envelope theorem and the above expressions, one obtains:

$$
\begin{aligned}
\frac{\partial \pi_{i}^{*}}{\partial c_{i}} & =x_{i} p^{\prime}(X) \Sigma_{j \neq i} \frac{\partial x_{j}}{\partial c_{i}}-x_{i}=x_{i} \Sigma_{j \neq i} \frac{p^{\prime}(X)+x_{j} p^{\prime \prime}(X)}{(n+1+E)\left(-p^{\prime}(X)\right)}-x_{i} \\
& =-x_{i}\left[\Sigma_{j \neq i} \frac{1+s_{j} E}{(n+1+E)}+1\right]=\frac{-x_{i}\left[2 n+\left(2-s_{i}\right) E\right]}{(n+1+E)} .
\end{aligned}
$$

With strategic substitutes (i.e., $\left.\alpha_{i} \leq 0\right), \partial \pi_{i}^{*} / \partial c_{i}<0$ follows immediately from (56) and (57). With strategic complements (i.e., $\alpha_{i}>0$ ), the first term or the sum in (57) becomes positive, which suggests the possibility of $\partial \pi_{i}^{*} / \partial c_{i}>$ $0 .{ }^{7}$ However, such possibility is prevented by the assumptions. Substituting $(55)$ into $\left[2 n+\left(2-s_{i}\right) E\right]$ in $(58)$, one has $\left[2 n+\left(2-s_{i}\right) E\right]>[2 n+2 E+1]=$ $2(E+n+1 / 2)$. Hence, assumption $i i$ ) (which implies (56)) leads to

$$
\frac{\left[2 n+\left(2-s_{i}\right) E\right]}{(n+1+E)}>0 .
$$

By (58), $\partial \pi_{i}^{*} / \partial c_{i}<0$ holds.

Q.E.D.

\section{References}

[1] Bertrand, J. (1883), Review of "Théorie mathématique de la richesse sociale" and "Recherches sur les principes mathématiques de la théorie des richesses," Journal des Savants (Paris), 499-508.

[2] Bulow, J., J. Geanakoplos, and P. Klemperer (1985), Multiproduct Oligopoly: Strategic Substitutes and Complements, Journal of Political Economy 93, 488511.

\footnotetext{
${ }^{7}$ Observe that such possibility is hindered by the second-order condition for $\pi$-max. By $\partial^{2} \pi_{i} / \partial^{2} x_{i}$ $=2 p^{\prime}(X)+x_{i} p^{\prime \prime}(X)<0 \Leftrightarrow \alpha_{i}=p^{\prime}(X)+x_{i} p^{\prime \prime}(X)<-p^{\prime}(X)$, the second-order condition for $\pi$-max places an upper bound on the size of $\alpha_{i}>0$, which reduces the possibility of $\partial \pi_{i}^{*} / \partial c_{i}>0$ in (57).
} 
[3] Cabral, L., and J. Villas-Boas (2001), Multiproduct Oligopoly and Bertrand Supertraps, Working Papers 01-04, Stern School of Business, Department of Economics, NYU.

[4] Deneckere, R., and C. Davidson (1985), Incentives to Form Coalitions with Bertrand Competition, Rand Journal of Economics 16, 473-486.

[5] Dixit, A. (1986), Comparative Statics for Oligopoly Theory, International Economic Review 27, 107-122.

[6] Février, P., and L. Linnemer (2004), Idiosyncratic Shocks in an Asymmetric Cournot Oligopoly, International Journal of Industrial Organization 22, 835-848.

[7] Goldberg, P.K. (1995), Product Differentiation and Oligopoly in International Markets: The Case of the US Automobile Industry, Econometrica 63, 891-951.

[8] Grossmann, V. (2003), Firm Size and Diversification: Asymmetric Multiproduct Firms under Cournot Competition, CESifo Working Paper No. 1047, University of Zurich.

[9] Harrington, J. (1987), Collusion in Multiproduct Oligopoly Games under a Finite Horizon, International Economic Review 28, 1-14.

[10] Johnson, J. P., and D. P. Myatt (2003), Multiproduct Quality Competition: Fighting Brands and Product Line Pruning, American Economic Review 93, $748-774$.

[11] Lal, R., and C. Matutes (1989), Price Competition in Multiproduct Duopolies, RAND Journal of Economics 20, 516-537.

[12] Novshek, W. (1985), On the Existence of Cournot Equilibrium, Review of Economic Studies 52, 85-98.

[13] Schelling, T. C. (1960), The Strategy of Conflicts. Cambridge, MA: Harvard University Press.

[14] Shapiro, C. (1989), Theories of Oligopoly Behavior, Ch. 6 in Handbook of Industrial Organization, R. Schmalensee and R. Willig, eds., Amsterdam: Elsevier Publishers.

[15] Shubik, M. (1980), Market Structure and Behavior. Cambridge, MA: Harvard University Press. 
[16] Smythe, D., and J. Zhao (2006), The Complete Welfare Effects of Cost Reductions in a Cournot Oligopoly, Journal of Economics 87, 181-193.

[17] Vives, X. (2005), Games with Strategic Complements: New Application to Industrial Organization, International Journal of Industrial Organization 23, 625-637.

[18] Zhang, A., and Y. Zhang (1996), Stability of a Cournot-Nash equilibrium: The Multiproduct Case, Journal of Mathematical Economics 26, 441-462.

[19] Zhao, J., and E. Howe (2004), Inverse Matrices and Merger Incentives from Bertrand Competition, Working Paper, Department of Economics, University of Saskatchewan. 